Publ. RIMS, Kyoto Univ.

25 (1989), 45-57

\title{
Endomorphisms of Certain Operator Algebras
}

\author{
By
}

\author{
Geoffrey L. PRICE*
}

\begin{abstract}
In this paper we study $C(X)$-endomorphisms of $C(X, \mathfrak{S}(\mathfrak{Y}))$, for $X$ a compact space. The local structure of these endomorphisms is analyzed. We prove the existence of a $C(X)$-shift, not all of whose fibers are shifts on $\mathfrak{B}(\mathfrak{E})$.
\end{abstract}

\section{$\S 1$. Introduction}

In [6] Kadison and Ringrose undertook a penetrating study of automorphisms of certain operator algebras. In particular, they showed that if $\mathfrak{A}$ is a $C^{*}$-algebra of operators on a Hilbert space $\mathfrak{S}$, then the condition $\|\alpha-\iota\|<2$ for an automorphism $\alpha$ implies that $\alpha$ lies in the connected component of the identity, $\Delta(\mathfrak{X})$, in $\operatorname{Aut}(\mathfrak{U})$. Moreover $\Delta(\mathfrak{X})$ is generated as a group by such automorphisms, and each element of $\Delta(\mathfrak{U})$ is permanently weakly inner, or $\pi$-inner. This latter property means that $\alpha$ is weakly inner in any (faithful) representation $\pi$ of $\mathfrak{A}$. If $\mathfrak{A}$ is the $C^{*}$-algebra $C(X, \mathfrak{B}(\mathfrak{S})$ ), where $X$ is a compact topological space, this property implies that $\alpha(f A)=f \alpha(A)$, for any scalar-valued function $f$ in $\mathfrak{A}$. Following the terminology of more recent papers, e.g., [8], we shall call such an automorphism a $C(X)$-automorphism.

In the case where $\mathfrak{S}$ is finite-dimensional, (i.e., $\mathfrak{V}(\mathfrak{S}) \cong M_{n}(\boldsymbol{C})$, for some $n$ ) Kadison and Ringrose give an example of a compact space $X[6$, Example $d]$ for which $\Delta(\mathfrak{U}) \subsetneq \operatorname{Inn}_{C(X)}(\mathfrak{U}) \subsetneq \operatorname{Aut}_{C(X)}(\mathfrak{U}) . \quad \operatorname{Inn}_{C(X)}(\mathfrak{X})$ is the group ofin ner $C(X)$ automorphisms, i.e., $\alpha$ is inner if there is a (uniformly continuous) unitaryvalued function $U$ on $X$ such that $\alpha=A d(U)$.

Motivated in part by [6], Lance, [7], and M.S. B. Smith, [14], studied the structure of the quotients of the various automorphism groups on $C(X, \mathfrak{B}(\mathfrak{S}))$, equipped with the norm topology, where $\mathfrak{S}$ is an infinite-dimensional Hilbert space. In this setting $\Delta(\mathfrak{X})$ and $\operatorname{Inn}_{C(X)}(\mathfrak{X})$ coincide, [7, Theorem 3.6], and the quotient group $\operatorname{Aut}_{C(X)}(\mathfrak{U}) / \Delta(\mathfrak{A})$ is shown to be isomorphic to $H^{2}(X, Z)[7$, Theorem 4.3]. A key result obtained by Lance to establish the above isomorphism is that $C(X)$-automorphisms of $C(X, \mathfrak{B}(\mathfrak{S}))$ are induced locally by norm

Communicated by H. Araki, April 12, 1988. Revised August 22, 1988.

* Department of Mathematics. United States Naval Academy, Annapolis, Maryland 21402, U.S. A.

** Supported in part by a grant from NSF. 
continuous unitary-valued functions, i. e., for $x \in X$ there is a neighborhood $N$ of $x$ and a unitary function $U \in C(N, \mathfrak{B}(\mathfrak{S}))$ such that $\alpha_{(C(N, \mathfrak{B}(\mathfrak{F}))}=A d(U)$. More recent papers (viz. $[8,9]$ ) have analyzed the structure of $C(X)$-automorphisms of separable continuous trace algebras; and, even more generally, of $C^{*}$-algebras of sections of bundles having fibres which are $C^{*}$-algebras.

In this note we introduce the notion of $C(X)$-shifts of $C(X, \mathfrak{B}(\mathfrak{S})$ ). (We shall assume throughout that $X$ is separable.) A *-endomorphism $\sigma$ of $C(X, \mathfrak{B}(\mathfrak{S}))$ which preserves the identity operator $\underline{I}$ (i.e., $I(x)=I$, for all $x$ ) and satisfies $\sigma(f A)=f \sigma(A)$ for $f \in C(X)$, shall be called a $C(X)$-endomorphism. Letting $A=\underline{I}$ we obtain $\sigma(f)=f$. If $\sigma$ satisfies the additional range property $\bigcap_{k \geq 1} \sigma^{k}(C(X, \mathfrak{B}(\mathfrak{S}))$ $=C(X)$, we say that it is a $C(X)$-shift. For $x$ a point, our definition coincides with that given by Powers [10] for a shift on $\mathfrak{B}(\mathfrak{S})$. In this setting, and under a technical assumption, Powers has shown that the Jones' index $[\mathfrak{B}(\mathfrak{S}): \sigma(\mathfrak{B}(\mathfrak{F}))]$ (cf. [5]) is a complete invariant for the outer conjugacy classes of shifts on $\mathfrak{B}(\mathfrak{S})$, [10, Theorem 2.4].

Although the general structure of $C(X)$-shifts on $C(X, \mathfrak{B}(\mathfrak{E}))$ is far from settled, our results here indicate an interesting interplay between the theory of $C(X)$-automorphisms on $C(X, \mathfrak{B}(\mathfrak{S})$ ) and the theory of shifts on $\mathfrak{B}(\mathfrak{S})$, (cf. [10, $12,13])$. In Section 2 , we show that $\left[\mathfrak{B}(\mathfrak{S}): \sigma_{x}(\mathfrak{B}(\mathfrak{E}))\right]$ is constant on connected component of $X$, where for $x \in X, \sigma_{x}$ is the *-endomorphism on $\mathfrak{B}(\mathfrak{S})$ induced by the equation $\sigma(A)(x)=\sigma_{x}(A(x)), A \in C(X, \mathfrak{B}(\mathfrak{Y}))$. In this section we also give an analogue of Lance's theorem on locally inner $C(X)$-automorphisms.

In Section 3 we present an example which shows that if $\sigma$ is a $C(X)$-shift, then not all of the induced endomorphisms, $\sigma_{x}$, of $\mathfrak{B}(\mathfrak{S})$ need to be shifts themselves. On the other hand, if each $\sigma_{x}$ is a shift it follows easily that $\sigma$ must be as well, Lemma 2.6 .

\section{§2. The Structure of $C(X)$-Endomorphisms}

Let $\sigma$ be a $C(X)$-endomorphism of $\mathfrak{A}$. Then $\sigma$ is an isometry of $\mathfrak{A}$, which fixes the center, $C(X)$. By a trivial adaptation of the proof of either [8, Lemma 1.4] or of [6, Example $d]$, we obtain the following local characterization of $\sigma$, which is essentially a restatement of [8, Lemma 1.4] for endomorphisms.

Lemma 2.1. Let $\sigma$ be a $C(X)$-endomorphisn on $\mathfrak{A}=C(X, \mathfrak{B}(\mathfrak{H}))$, where $X$ is a compact space.

(i) If $A_{1}, A_{2} \in \mathfrak{A}$ and $A_{1}(x)=A_{2}(x)$ for some $x \in X$, then $\sigma\left(A_{1}\right)(x)=\sigma\left(A_{2}\right)(x)$.

(ii) For any compact set $Y$ of $X, \sigma$ induces a (unit-preserving) *-endomorphism $\sigma_{Y}$ of $C(Y, \mathfrak{B}(\mathfrak{Y}))$ such that $\sigma_{Y}\left(A_{\mid Y}\right)=\sigma(A)_{\mid Y}$ for $A \in \mathfrak{B}$.

We write $\sigma_{x}$ for the unique *-endomorphism of $\mathfrak{B}(\mathfrak{g})$ which satisfies $\sigma(A)(x)$ $=\sigma_{x}(A(x)), x \in X, A \in \mathfrak{Y}$. We shall write $\sigma=\bigoplus_{x \in X} \sigma_{x}$. Observe that the *-endomor- 
phisms $\sigma_{x}$ vary continuously with $x$ in the norm topology. For if $A \in \mathfrak{B}(\mathfrak{S})$, then $\sigma(\underline{A}) \in C(X, \mathfrak{B}(\mathfrak{S}))$, where $\underline{A}$ is the constant function $\underline{A}(y)=A$, for all $y \in X$, so that given $x \in X$ and $\varepsilon>0$, there is a neighborhood $N$ of $x$ such that $\| \sigma(\underline{A})(y)$ $-\sigma^{\prime}(\underline{A})(x) \|<\varepsilon$, for $y \in N$. But $\|\sigma(\underline{A})(y)-\sigma(\underline{A})(x)\|=\left\|\sigma_{x}(A)-\sigma_{y}(A)\right\|$, by Lemma 1. Hence we have the following (cf. [8, Lemma 1.6]).

Lemma 2.2. Let $\sigma=\bigoplus_{x \in X} \sigma_{x}$ be a *endomorphisin on $C(X, \mathfrak{B}(\mathfrak{S}))$. Then the

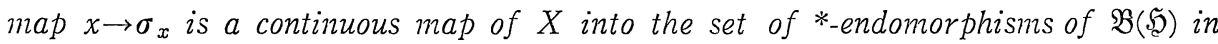
the topology of pointwise norm convergence.

Now suppose $\alpha$ is a *-endomorphism of $\mathfrak{B}(\mathfrak{S})$ which preserves the identity. Then $\alpha\left(\mathfrak{B}(\mathfrak{E})\right.$ ) is a subfactor of $\mathfrak{B}(\mathfrak{F})$, isomorphic to $\mathfrak{B}(\mathfrak{E})$. Moreover, $\alpha(\mathfrak{B}(\mathfrak{F}))^{\prime}$ is a type $I_{n}$ subfactor $N$ of $\mathfrak{B}(\mathfrak{S})$, for some $n$ (possibly $\infty$ ) and $\mathfrak{B}(\mathfrak{S})$ is generated by $N$ and $\alpha(\mathfrak{B}(\mathfrak{S}))[1, \mathrm{I} .8 .2$, Corollary 3]. Using the identification $\mathfrak{B}(\mathfrak{S}) \cong N \otimes$ $\alpha(\mathfrak{B}(\mathfrak{S}))$, it is clear that if $e \in \mathfrak{B}(\mathfrak{S})$ is a rank one projection, then $\alpha(e)$ is a projection in $\mathfrak{B}(\mathfrak{S})$ of rank $n$. Another way to characterize the "size" of $\alpha(\mathfrak{B}(\mathfrak{S})$ ) in $\mathfrak{B}(\mathfrak{F})$ is to observe that $n^{2}=[\mathfrak{B}(\mathfrak{F}): \alpha(\mathfrak{B}(\mathfrak{E}))]$, the Jones index (cf. [5]).

Definition 2.1. Let $\alpha$ be a $*$-endomorphism of $\mathfrak{B}(\mathfrak{S})$. Then $\alpha$ has index $n^{2}$ if $\alpha(\mathfrak{B}(\mathfrak{S}))^{\prime}$ is a type $I_{n}$ factor.

Proposition 2.3. Let $\sigma=\oplus \sigma_{x}$ be a $C(X)$-endomorphism of $\mathfrak{A}$. The index of the endomorphisms $\sigma_{x}$ is constant on connected components of $X$.

Proof. Let $e \in \mathfrak{B}(\mathfrak{S})$ be a rank one projection, then the mapping $x \rightarrow \sigma(\underline{e})(x)=$ $\sigma_{x}(e)$ is uniformly continuous. Given $x \in X$, let $N$ be a neighborhood of $x$ such that for $y \in N,\left\|\sigma_{y}(e)-\sigma_{x}(e)\right\|<1$. Then [3, Lemma 1.8] there is a partial isometry with support projection $\sigma_{x}(e)$ and range projection $\sigma_{y}(e)$, so the projections $\sigma_{y}(e)$, $\sigma_{x}(e)$ have the same rank. Hence $x \rightarrow \operatorname{rank}\left(\sigma_{x}(e)\right)$ is a continuous function from $X$ to $N \cup\{\infty\}$, and the result follows.

In what follows we shall make the assumption that $X$ is connected. Fix $x \in X$; then for any $y \in X, \sigma_{x}, \sigma_{y}$ have the same index. We shall also assume that this index is finite. We may then construct a unitary operator $U_{y} \in \mathfrak{B}(\mathfrak{S})$ such that $A d\left(U_{y}\right) \circ \sigma_{x}=\sigma_{y}$. In fact, choosing matrix units $\left\{e_{\imath j}: i, j \in N\right\}$ for $\mathfrak{B}(\mathfrak{S})$, we set

$$
U_{y}=\sum_{i=1}^{\infty} \sigma_{y}\left(e_{\imath 1}\right) V_{y} \sigma_{x}\left(e_{1 \imath}\right),
$$

as in [10, Theorem 2.4], where $V_{y} \in \mathfrak{B}(\mathfrak{g})$ is a partial isometry from $\sigma_{x}\left(e_{11}\right)$ to $\sigma_{y}\left(e_{11}\right)$. Observe that if $U_{y}^{\prime}$ is any other unitary such that $A d\left(U_{y}^{\prime}\right) \circ \sigma_{x}=\sigma_{y}$, then $U_{y}^{*} U_{y}^{\prime} \in \sigma_{x}(\mathfrak{B}(\mathfrak{G}))^{\prime}$.

Lemma 2.4. Let $N$ be a neighborhood of $x \in X$, where $X$ is connected. Suppose there is a uniformly continuous mapping $y \rightarrow V_{y}$ from $N$ to partial isometries $V_{y}$ 
with support projection $\sigma_{x}\left(e_{11}\right)$ and range projection $\sigma_{y}\left(e_{11}\right)$. Then there is $a$ strongly continuous mapping $y \rightarrow U_{y}$ on $N$ where the $U_{y}$ are unitary operators satisfying (1).

Proof. Suppose $\left\{f_{i}: i \in N\right\}$ is an orthonormal basis for $\mathfrak{H}$ such that for each $i, j \in N$, either $\sigma_{x}\left(e_{j j}\right) f_{i}=0$, or $f_{\imath}$. Let $U_{y} \in \mathfrak{B}(\mathfrak{g})$ be defined as in (1). Since $\left\|U_{y}\right\|=1$, it suffices to show that $y \rightarrow U_{y} f$ is continuous for $f$ a finite linear combination of elements of the basis; for the same reason, we may assume that $f=f_{i}$, for some $i \in \boldsymbol{N}$. Choose $j$ such that $\sigma_{x}\left(e_{j j}\right) f=f$; then $U_{y} f$ $=\sigma_{y}\left(e_{j j}\right) V_{y} \varphi$, where $\varphi=\sigma_{x}\left(e_{12}\right) f$. But $y \rightarrow \sigma_{y}\left(e_{\jmath \jmath}\right)$ and $y \rightarrow V_{y}$ are uniformly continuous for $y \in N$, so the mapping $y \rightarrow \sigma_{y}\left(e_{j}\right) V_{y} \varphi$ is also continuous on $N$.

Proposition 2.5. Let $x \in X$. There is a neighborhood $N$ of $x$ such that a uniformly continuous mapping $y \rightarrow V_{y}$ exists from $N$ to partial isometries $V_{y}$ with support projection $\sigma_{x}\left(e_{11}\right)$ and range projection $\sigma_{y}\left(e_{11}\right)$.

Proof. For any $\gamma$ such that $0<\gamma<1$, let $N$ be a neighborhood of $x$ such that $\left\|\sigma_{y}\left(e_{11}\right)-\sigma_{x}\left(e_{11}\right)\right\|<\gamma$, for all $y \in N$. Let $P$ (respectively, $Q$ ) be the projection given by $P(y)=\sigma_{x}\left(e_{11}\right)$ (resp., $Q(y)=\sigma_{y}\left(e_{11}\right)$ ) for all $y \in \bar{N}$. Then $\|P-Q\|$ $<1$, so by [3, Lemma 1.8] there exists a unitary $U \in C(\bar{N}, \mathfrak{B}(\mathfrak{S}))$ such that $Q=$ $U P U^{*}$. Setting $V=U P, y \rightarrow V(y)$ gives the desired mapping.

Corollary. There are, for each $x \in X$, a neighborhood $N$ of $x$, and unitary operators $U_{y} \in \mathfrak{B}(\mathfrak{T})$ such that the mapping $y \rightarrow U_{y}$ is strongly continuous for $y \in N$.

In what follows we shall show that under some restrictions the preceding result may be strengthened in the sense that the $U_{y}$ may be chosen to vary continuously in norm for $y \in N$, (Theorem 2.8). This result is inspired by a similar result obtained by Lance, [7], for $C(X)$-automorphisms, which was described in Section 1. We have also adapted some of the techniques of that proof.

Definition 2.2. Let $\sigma=\bigoplus \sigma_{x}$ be a $C(X)$-endomorphism of $C(X, \mathfrak{B}(\mathfrak{T}))$, and let $\rho$ be a state of $\mathfrak{B}(\mathfrak{S})$. We say that $\rho$ is $\sigma$-invariant if $\rho \circ \sigma_{x}=\rho$ for all $x \in X$.

Throughout the remainder of this section we assume the existence of a pure normal state $\rho$ on $\mathfrak{B}(\mathfrak{S})$ which is $\sigma$-invariant. We shall also assume that $\sigma_{x}$ is a shift on $\mathfrak{B}(\mathfrak{S})$, for each $x \in X$. In this situation, we have the following structure theorem of Powers, [10, Theorem 2.3].

Theorem $\mathbf{P}$. Suppose $\alpha$ is a shift of $\mathfrak{B}(\mathfrak{S})$. If there is an $\alpha$-invariant pure normal state on $\mathfrak{B}(\mathfrak{S})$, then there are mutually commuting, isomorphic type $I$ subfactors $B_{1}, B_{2}, \cdots$ of $\mathfrak{B}(\mathfrak{S})$ such that

(i) $\mathfrak{B}(\mathfrak{F})$ is the weak closure of $B_{1} \otimes B_{2} \otimes \cdots$,

(ii) $\alpha\left(B_{k}\right)=B_{k+1}$ for $k \in N$, and 
(iii) $\rho$ is a symmetric product state with respect to the factorization in (i).

Lemma 2.6. Suppose $\sigma=\bigoplus \sigma_{x}$ is a $C(X)$-endomorphism such that $\sigma_{x}$ is a shift of $\mathfrak{B}(\mathfrak{S})$, for all $x \in X$. Then $\sigma$ is a $C(X)$-shift.

Proof. Let $A \in C(X, \mathfrak{B}(\mathfrak{g}))$ lie in the range of $\sigma^{k}$, for all $k \in N$. For each $k$, let $A_{k}$ satisfy $\sigma^{k}\left(A_{k}\right)=A$. If $x \in X, \sigma_{x}^{k}\left(A_{k}(x)\right)=\sigma^{k}\left(A_{k}\right)(x)=A(x)$, so $A(x) \in$ $\bigcap_{k \geq 1} \sigma_{x}^{k}(\mathfrak{B}(\mathfrak{S}))$ is a scalar multiple of the identity, and therefore $A \in C(X)$. Hence $\sigma$ is a shift.

Remark. The converse of the lemma is not true, in general. This is the principal result of the following section.

The following technical lemma will be helpful in proving the main result of this section, Theorem 2.8, (cf. [7, Lemma 3.2]).

Lemma 2.7. Suppose, for some $x \in X$ there are a neighborhood $N$ of $x$ and unitary operators $U_{y}$ for each $y \in N$ such that $U_{y} \rightarrow I$ strongly, but not uniformly, as $y \rightarrow x$. Then there are an orthonormal sequence $\left\{h_{m}\right\}$ of vectors of $\mathfrak{S}$, a sequence $\left\{y_{m}\right\}$ in $N$, and $a \delta>0$ such that

(i) $y_{m} \rightarrow x$

(ii) $\left\|U_{y_{n}} h_{m}-h_{m}\right\|<\delta /\left(n 2^{n+1}\right)$, for $m<n$,

(iii) $\left\|U_{y_{m}} h_{m}-h_{m}\right\|>\delta / 2$.

Proof. Under the hypotheses, there are a sequence $\left\{\varphi_{m}\right\}$ of unit vectors in $\mathfrak{F}$, elements $x_{n} \in N$, and $\delta>0$ such that (a) $\left\|U_{x_{n}} \varphi_{m}-\varphi_{m}\right\|<\delta /\left(n 2^{n+1}\right)$, for $m<n$, and (b) $\left\|U_{x_{n}} \varphi_{n}-\varphi_{n}\right\|>\delta$. Fix $n$, and suppose orthonormal vectors $h_{1}, h_{2}, \cdots, h_{n}$ have been chosen in the subspace of $\mathfrak{H}$ spanned by the $\varphi_{m}$ 's, and elements $y_{1}$, $y_{2}, \cdots, y_{n}$ have been selected from the sequence $\left\{x_{m}\right\}$ such that

(1) $\left\|U_{y_{p}} h_{m}-h_{m}\right\|<\delta /\left(p 2^{p+1}\right), \quad$ for $1 \leqq m<p \leqq n$,

(2) $\left\|U_{y_{p}} h_{p}-h_{p}\right\|>\delta / 2$, for $1 \leqq p \leqq n$.

Choose an element $y \in\left\{x_{m}\right\}$ such that $\left\|U_{y} h_{p}-h_{p}\right\|<\delta /\left((n+1) 2^{n+1}\right)$, for $1 \leqq p \leqq n$. Writing $\varphi^{\prime}=\varphi_{y}$, then $\left\|U_{y} \varphi^{\prime}-\varphi^{\prime}\right\|>\delta$. Let $\varphi=\sum_{k=1}^{n}\left(\varphi^{\prime}, h_{k}\right) h_{k}$, and let $\phi=\varphi-\varphi^{\prime}$. Then $\phi \perp h_{k}$, for $k \leqq n$, and

$$
\begin{aligned}
\delta<\left\|U_{y} \varphi^{\prime}-\varphi^{\prime}\right\| & \leqq\left\|U_{y} \psi-\psi\right\|+\left\|U_{y} \varphi-\varphi\right\| \\
& \leqq\left\|U_{y} \psi-\psi\right\|+\left\|\sum_{k=1}^{n}\left(\varphi^{\prime}, h_{k}\right)\left(U_{y} h_{k}-h_{k}\right)\right\| \\
& \leqq\left\|U_{y} \psi-\psi\right\|+\left[\max _{1 \leqq k \leqq n}\left\{\left|\left(\varphi^{\prime}, h_{k}\right)\right|\right\}\right]\left(n \delta /\left((n+1) 2^{n+1}\right)\right) \\
& \leqq\left\|U_{y} \psi-\psi\right\|+\delta /\left(2^{n+1}\right) .
\end{aligned}
$$


Hence $\delta / 2<\delta-\left(\delta / 2^{n+1}\right)<\left\|U_{y} \psi-\phi\right\| \leqq\left\|U_{y} \psi-\phi\right\| /\|\psi\|$. Setting $y_{n+1}=y$, and $h_{n+1}=$ $\phi /\|\psi\|$, (i) and (ii) hold for $k=1,2, \cdots, n+1$. The result now follows by induction.

Using the preceding results we may now improve Corollary 2.5 as follows. The main idea of the proof (i.e., the construction of the operator $V$ ), is inspired by a similar construction in [7, Lemma 3.2].

Theorem 2.8. Let $\sigma=\bigoplus_{x \in X} \sigma_{x}$ be a $C(X)$-shift of $C(X, B(\mathfrak{S}))$ on a connected space $X$, for which each $\sigma_{x}$ is a shift of finite index on $\mathfrak{B}(\mathfrak{K})$. Suppose there is a $\sigma$-invariant pure normal state $\rho$ on $\mathfrak{B}(\mathfrak{S})$. Then for each $x \in X$, there is a neighborhood $N$ of $x$ such that

(i ) $\sigma_{y}=A d\left(U_{y}\right) \circ \sigma_{x}$, for $y \in N$, and

(ii) the $U_{y}$ vary continuously in norm for $y \in N$.

Proof. From Corollary 2.5 we may choose for each $x \in X$ a neighborhood $N$ of $x$ and unitary operators $V_{y}$ for $y \in N$, which satisfy (i) and which vary continuously in the strong topology. For $A \in \mathfrak{B}(\mathfrak{H}), \sigma_{x}(A)=V_{x} \sigma_{x}(A) V_{x}^{*}$, so $V_{x} \in$ $\sigma_{x}(\mathfrak{B}(\mathfrak{S}))^{\prime}=B_{1}$, where we use the notation of Theorem P. Taking $U_{y}=V_{y} V_{x}^{*}$, (i) still holds and $U_{x}=I$.

From Theorem $\mathrm{P}$ we may identify $\mathfrak{B}(\mathfrak{g})$ as the weak closure of the tensor product $B_{1} \otimes B_{2} \otimes \cdots$. By making the identification $B_{k}=B_{j}$, for all $k, j$ each $B_{k}$ may be viewed as acting on the same $n$-dimensional Hilbert space $\mathfrak{S}_{0}$ with orthonormal basis $\left\{f_{1}, \cdots, f_{n}\right\}$. If $E_{k}$ is the minimal projection of $B_{k}$ for which $\rho\left(E_{k}\right)=1$, then $\sigma_{x}\left(E_{k}\right)=E_{k+1}$, and we may assume $E_{k} f_{1}=f_{1}$, for all $k$. We then view $\mathfrak{H}$ as the Hilbert space tensor product $\otimes \mathfrak{S}_{0}$, having orthonormal basis the vectors $f_{j_{1}} \otimes f_{j_{2}} \otimes \cdots$ for which all but finitely many indices $j_{k}$ are 1(see [4]).

If $\left\|U_{y}-I\right\| \nrightarrow 0$ as $y \rightarrow x$ in $N$, there is an orthonormal sequence $\left\{h_{m}\right\}$ in $\mathfrak{S}$ which satisfies the conditions of Lemma 2.7. From the finite-dimensionality of $\mathfrak{S}_{0}$ we may assume that there is a unit vector $f \in \mathfrak{V}_{0}$ such that each $h_{i}$ has the form $h_{\imath}=f \otimes \varphi_{i}$, where the $\varphi_{i}$ 's are orthonormal. Viewing the $\varphi_{i}$ as elements of $\mathfrak{W}$, let $e_{i}$ be the rank one projections satisfying $e_{\imath} \varphi_{i}=\varphi_{i}$. Define $e_{i+1} \otimes e_{i}$ to be the rank one operator $\left(e_{\imath+1} \otimes e_{2}\right) \varphi=\left(\varphi, \varphi_{i}\right) \varphi_{i+1}$. Then the sum $\sum_{i=1}^{\infty} e_{i+1} \otimes e_{i}$ converges strongly to a partial isometry $V$ in $\mathfrak{B}(\mathfrak{H})$. Moreover, for a vector of the form $f \otimes \varphi \in \mathfrak{L}, \sigma_{x}(V)(f \otimes \varphi)=f \otimes V \varphi$.

Let $\varepsilon=\delta / 4$ (for $\delta$ as in Lemma 2.7). By the lemma there is an $m \in \boldsymbol{N}$ and a $y=y_{m+1} \in N$ such that $\left\|U_{y} h_{m}-h_{m}\right\|<\varepsilon / 2$ and $\left\|U_{y} h_{m+1}-h_{m+1}\right\|>2 \varepsilon$. Then $\left\|\sigma_{x}(V) U_{y} h_{m}-\sigma_{x}(V) h_{m}\right\|<\varepsilon / 2$, and

$$
\begin{aligned}
\sigma_{x}(V) h_{m} & =\sigma_{x}(V)\left(f \otimes \varphi_{m}\right) \\
& =f \otimes V \varphi_{m}
\end{aligned}
$$




$$
=f \otimes \varphi_{m+1} .
$$

On the other hand, $\left\|U_{y} \sigma_{x}(V) h_{m}-f \otimes \varphi_{m+1}\right\|=\left\|U_{y}\left(f \otimes \varphi_{m+1}\right)-f \otimes \varphi_{m+1}\right\|>2 \varepsilon$, so by combining we have $\left\|\sigma_{x}(V) U_{y} h_{m}-U_{y} \sigma_{x}(V) h_{m}\right\|>\varepsilon$. But

$$
\begin{aligned}
\left\|\sigma_{y}(V)-\sigma_{x}(V)\right\| & =\left\|U_{y} \sigma_{x}(V) U_{y}^{*}-\sigma_{x}(V)\right\| \\
& =\left\|U_{y} \sigma_{x}(V)-\sigma_{x}(V) U_{y}\right\| \\
& \geqq\left\|U_{y} \sigma_{x}(V) h_{m}-\sigma_{x}(V) U_{y} h_{m}\right\|>\varepsilon,
\end{aligned}
$$

so that $\sigma_{y}(V) \nrightarrow \sigma_{x}(V)$ in norm for $y$ in the sequence $\left\{y_{m}\right\}$, a contradiction, since $\sigma(\underline{V}) \subseteq C(X, \mathfrak{B}(\mathfrak{S}))$, where $\underline{V}(y)=V$, for all $y$. By the contradiction, the $U_{y}$ vary continuously in norm in $N$, and we are done.

Remark. If $X$ is connected, fix an element $x \in X$, and let $\left\{N_{\imath}\right\}_{i \in \mathcal{I}}$ be a finite open cover of $X$ with uniformly continuous functions $\left\{U_{i}\right\}_{\imath \in \mathcal{J}}$ implementing $\sigma$ in the sense that for $y \in N_{i}, \sigma_{y}=U_{i}(y) \sigma_{x}() U_{i}(y)^{*}$. On $N_{i j}=N_{i} \cap N_{j}, \sigma_{y}=$ $A d\left(U_{\imath}(y)\right) \circ \sigma_{x}=A d\left(U_{j}(y)\right) \circ \sigma_{x}$, so $U_{\imath}(y) U_{j}(y)^{*} \in \sigma_{x}(\mathfrak{B}(\mathfrak{g}))^{\prime}=B_{1}$. If $\gamma$ denotes the determinant on $B_{1}$, then let $\lambda_{i j}(y)=\gamma\left(U_{\imath}(y) U_{j}(y)^{*}\right)$. Then on $N_{i j k}=N_{i} \cap N_{j} \cap N_{k}$, $\lambda_{i j} \lambda_{j k}=\gamma\left(U_{i} U_{j}^{*}\right) \gamma\left(U_{j} U_{k}^{*}\right)=\gamma\left(U_{2} U_{k}^{*}\right)=\lambda_{i k}$, so the $\lambda_{i j}$ 's define a one-cocycle of $\left\{N_{i}\right\}$ whose values lie in the sheaf of germs of continuous functions from $X$ to $\Pi^{1}$ (cf. [7, Lemma 4.1]). Arguing as in [8], if $\left\{M_{p}\right\}$ is another cover, with associated unitary functions $V_{p}(y)$ on $M_{p}$, then passing to a common refinement of $\left\{N_{i}\right\}$ and $\left\{M_{p}\right\}$ we may assume that the two covers coincide. On $N_{i}$, let $\Delta_{i}$ be the continuous function determined by $\gamma\left(V_{i}\right)=\Delta_{i} \gamma\left(U_{i}\right)$. If $\left\{\mu_{i j}\right\}$ define the one-cocycle of $\left\{N_{\imath}\right\}$ for $\left\{V_{i}\right\}$ in the sense that $\mu_{i j}(y)=\gamma\left(V_{\imath}(y) V_{j}(y)^{*}\right)$, then

$$
\mu_{\imath \jmath}=\gamma\left(V_{i} V_{j}^{*}\right)=\Delta_{\imath} \Delta_{j}^{-1} \gamma\left(U_{\imath} U_{j}^{*}\right)=\Delta_{\imath} \Delta_{j}^{-1} \lambda_{i j}
$$

so that $\mu_{\imath j}, \lambda_{\imath \jmath}$ differ by a coboundary. Hence they define the same element of $H^{1}\left(X, \Pi^{1}\right)$. It would be interesting to pursue these results, which are analogous to the automorphism case, to determine more information about the structure of the unitaries $\left\{U_{\imath}\right\}$ implementing $\sigma$.

\section{§3. An Example of a Shift}

In this section we present a counterexample to the converse of Lemma 2.6. We verify the existence of a $C(X)$-shift $\sigma=\bigoplus_{x \in X} \sigma_{x}$, where $X$ is a closed interval

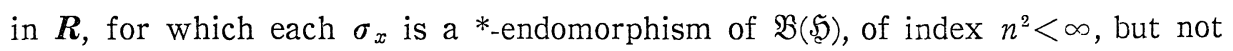
all $\sigma_{x}$ 's are shifts on $\mathfrak{B}(\mathfrak{Y})$.

We fix some notation. As above, let $B_{k}, k \in N \cup\{0\}$, be copies of $M_{n}(\boldsymbol{C})$ acting on $\boldsymbol{C}^{n}$, with orthonormal basis $\left\{\Omega_{1}, \cdots, \Omega_{n}\right\}$. Let $\left\{e_{\imath \jmath}^{k}: 1 \leqq i, j \leqq n\right\}$ be matrix units for $B_{k}$ satisfying $e_{\imath \jmath}^{k} \Omega_{p}=\delta_{\jmath p} \Omega_{\imath}$. For convenience, write $\Omega=\Omega_{1}$. $\mathfrak{U}$ is the UHF algebra $\bigotimes_{k \geq 0} B_{k}$ of type $n^{\infty}$. This algebra acts on the Hilbert 
space $\mathfrak{S}$ having an orthonormal basis consisting of all vectors of the form $\Omega_{j} \otimes \Omega_{i_{1}} \otimes \Omega_{i_{2}} \otimes \cdots$, where all but finitely many of the $\Omega_{i_{k}}$ are $\Omega$. Under this action, $B(\mathfrak{S})$ is the weak operator closure of $\mathfrak{X}$. Let $\omega$ be the vector state $\omega_{\Omega}$, on $\mathfrak{A}$, where $\Omega^{\prime}=\Omega \otimes \Omega \otimes \cdots$. Then $\omega$ is a pure symmetric product state, $\underset{k \geq 0}{\otimes} \omega_{k}$, on $\mathfrak{A}$, where

$$
\omega_{k}\left(e_{i j}^{k}\right)=\delta_{i j} \delta_{\imath 1}, \quad k \in N \cup\{0\} .
$$

Let $\alpha$ be the shift on $\left(\bigotimes_{k \geqq 1} B_{k}\right)^{\prime \prime}$ given by $\alpha\left(e_{i j}^{k}\right)=e_{i j}^{k+1}$. Define a *-endomorphism $\sigma_{0}=\imath \otimes \alpha$ on $\mathfrak{B}(\mathfrak{S})$, i. e., $\sigma_{0}(A)=A$ for $A \in B_{0}$, and $\sigma_{0}(A)=\alpha(A)$ for $A \in$ $\left(\otimes_{k=1} B_{k}\right)^{\prime \prime}$. It is straightforward to verify that $n^{2}=\left[\mathfrak{B}(\mathfrak{S}): \sigma_{0}(\mathfrak{B}(\mathfrak{S}))\right]$. Moreover $\sigma_{0}$ is not a shift on $\mathfrak{B}(\mathfrak{S})$; in fact, it is easy to verify that $B_{0}=\bigcap_{k \geqq 1} \sigma_{0}^{k}(\mathfrak{B}(\mathfrak{S}))$.

As in [11] we define an action on $\mathfrak{A}$ of the discrete group $S(\infty)$ of permutations of $N \cup\{0\}$ fixing all but finitely many elements. We define this action by setting $(r s) e_{p q}^{k}(r s)=e_{p q}^{k}$, if $k \neq r$ and $k \neq s$, and $(r s) e_{p q}^{r}(r s)=e_{p q}^{s}$, for transpositions $(r s)$ in $S(\infty)$. It is straightforward to show that $(r s)$ is inner; in fact, we may take $(r s)$ to be the (self-adjoint) unitary element $\sum_{p=1} \sum_{q=1} e_{p q}^{r} e_{q p}^{s}$ (cf. [11]).

Definition 3.1. Let $T$ be the transposition (01). For $t \in \boldsymbol{R}$, let $U_{t}$ be the unitary operator $e^{-i t} \exp (i t T)$. More generally for $k \in N$, let $T_{k}=(0 k)$, and $U_{k, t}$ $=e^{-i t} \exp \left(i t T_{k}\right)$.

Lemma 3.1. The following conditions hold for the operators defined as above:

(i ) $T_{k}=\sigma_{0}^{k}(T), \quad$ for $k \in \boldsymbol{N} \cup\{0\}$,

(ii) $U_{k, t}=e^{-i t}\left\{\cos (t)+\mathrm{i} \sin (t) T_{k}\right\}$,

(iii) $\omega \circ A d\left(U_{k, t}\right)=\omega$

(iv) $\omega \circ \sigma_{0}=\omega$

(v) $\omega \circ A d\left(U_{k, t}\right) \circ \sigma_{0}=\omega$.

Proof. (i) and (ii) are obvious. To show (iii) observe that $T_{k} \Omega^{\prime}=\Omega^{\prime}$, so $\exp \left(i t T_{k}\right)=e^{i t} \Omega^{\prime}$. Hence $\omega\left(U_{k, t} A U_{k, t}^{*}\right)=\left\langle U_{k, t} A U_{k, t}^{*} \Omega^{\prime}, \Omega^{\prime}\right\rangle=\left\langle\mathrm{A} \Omega^{\prime}, \Omega^{\prime}\right\rangle=\omega(A), A \in$ $\mathfrak{B}(\mathfrak{S})$. To verify (iv) observe that for $k \in N, \omega\left(\sigma_{0}\left(e_{i j}^{k}\right)\right)=\omega\left(e_{i j}^{k+1}\right)=\omega\left(e_{i j}^{k}\right)$, by (2). Sicne $\omega$ is a product state the preceding equation shows that $\omega$ is $\sigma_{0}$-invariant. (v) follows from (iii) and (iv).

Now let $x=[-\pi / 4, \pi / 4]$, and define $\sigma^{\prime}=\bigoplus_{t \in x} \sigma_{t}^{\prime}$, where $\sigma_{t}^{\prime}=\sigma_{0}$ for all $t$. Clearly, $\sigma^{\prime}$ is a $C(X)$-endomorphism of $C(X, \mathfrak{B}(\mathfrak{S}))$. Define $\sigma=\bigoplus_{t \in x} \sigma_{t}$, where $\sigma_{t}=$ $A d\left(U_{t}\right) \circ \sigma_{t}^{\prime}$. Since $U=\oplus U_{t} \in C(X, \mathfrak{B}(\mathfrak{g})), \sigma=A d(U) \circ \sigma^{\prime}$ is also a $C(X)$-endomorphism. We shall show that $\sigma$ is in fact a $C(X)$-shift. This is achieved through the series of lemmas below.

Definition 3.2. For $k \in \boldsymbol{N} \cup\{0\}$, and $t \in \boldsymbol{R}$, let $\Gamma_{k, t}$ be the unitary operator 
$U_{t} \sigma_{0}\left(U_{t}\right) \cdots \sigma_{0}^{k}\left(U_{t}\right)=U_{1, t} U_{2, t} \cdots U_{k+1, t}$.

It is straightforward to show that since $\sigma_{t}=A d\left(U_{t}\right) \circ \sigma_{0}$,

for $A \in \mathfrak{B}(\mathfrak{S})$.

$$
\sigma_{t}^{k+1}(A)=\Gamma_{k, t} \sigma_{0}^{k+1}(A) \Gamma_{k, t}^{*},
$$

Lemma 3.2. For $t \in X$, and for $k \in N \cup\{0\},\left[\sigma_{t}^{k+1}(\mathfrak{B}(\mathfrak{S}))\right]^{\prime}=\Gamma_{k, t}\left\{B_{1}, \cdots\right.$, $\left.B_{k+1}\right\}^{\prime \prime} \Gamma_{k, t}^{*}$.

Proof. Since $\sigma_{0}=\iota \otimes \alpha$, and since $\left[\alpha^{k}\left(\bigotimes_{j \geq 1} B_{\jmath}\right)\right]^{\prime} \cap\left(\bigotimes_{j \geq 1} B_{j}\right)=\left\{B_{1}, \cdots, B_{k}\right\}^{\prime \prime}$, by $\left[10, \quad\right.$ Lemma 2.1], $\sigma_{0}^{k}(\mathfrak{B}(\mathfrak{S}))^{\prime}=\left\{B_{1}, \cdots, B_{k}\right\}^{\prime \prime} . \quad$ Then by $(3), \quad\left[\sigma_{t}^{k+1}(\mathfrak{B}(\mathfrak{Y}))\right]^{\prime}=$ $\left(\Gamma_{k, t} \sigma_{0}^{k+1}(\mathfrak{B}(\mathfrak{S})) \Gamma_{k, t}^{*}\right)^{\prime}=\Gamma_{k, t}\left[\sigma_{0}^{k+1}(\mathfrak{B}(\mathfrak{S}))\right]^{\prime} \Gamma_{k, t}^{*}=\Gamma_{k, t}\left\{B_{1}, \cdots, B_{k+1}\right\}^{\prime \prime} \Gamma_{k, t}^{*}$.

Lemma 3.3. For $t \in X$, and for $k \in N, \sigma_{t}^{k+1}(\mathfrak{B}(\mathfrak{S}))=\left\{\Gamma_{k, t} B_{0} \Gamma_{k, t}^{*}, B_{k+2}, B_{k+3}\right.$, ... $\}^{\prime \prime}$.

Proof. Clearly $\sigma_{0}^{k+1}(\mathfrak{B}(\mathfrak{S}))=\left\{B_{0}, B_{k+2}, \cdots\right\}^{\prime \prime}$. Then $\sigma_{t}^{k+1}(\mathfrak{B}(\mathfrak{S}))=\left\{\Gamma_{k, t} B_{0} \Gamma_{k, t}^{*}\right.$, $\left.\Gamma_{k, t} B_{k+2} \Gamma_{k, t}^{*}, \cdots\right\}^{\prime \prime}$. But $\Gamma_{k, t} \in\left\{B_{0}, B_{1}, \cdots, B_{k+1}\right\}^{\prime \prime}$, so commutes with $B_{k+j}$, for $j>1$, so $\Gamma_{k, t} B_{k+j} \Gamma_{k, t}^{*}=B_{k+j}$.

By $\Phi_{k}, k \in N$, we shall denote the conditional expectation of the vector state $\omega$ onto $\bigotimes_{j=0}^{k} B_{j}$. We may define $\Phi_{k}$ as follows (cf. [2, Lemma 2]). Let $\left\{E_{p q}\right\}$ be matrix units for $\bigotimes_{j=0}^{k} B_{j}$. Each $A \in \mathfrak{B}(\mathfrak{L})$ has a unique decomposition of the form $\sum E_{p q} A_{p q}$, where $A_{p q} \in\left\{B_{k+1}, \cdots\right\}^{\prime \prime}$. Then $\Sigma \omega\left(A_{p q}\right) E_{p q}=\Phi_{k}(A)$.

Lemma 3.4. The sequence $\left\{\Phi_{k}(A): k \in N\right\}$ converges weakly to $A$, for all $A \in \mathfrak{B}(\mathfrak{S})$.

Proof. Since $\|A\| \geqq\left\|\Phi_{k}(A)\right\|$ for all $k \in N$, it suffices to show $\left\langle\Phi_{k}(A) \xi, \eta\right\rangle$ converges to $\langle A \xi, \eta\rangle$ for basis vectors $\xi=\bigotimes_{i \geq 0} \Omega_{p_{\imath}}, \eta=\bigotimes_{i \geq 0} \Omega_{q_{i}}$. For $N$ sufficiently large, $\Omega_{p_{k}}=\Omega_{q_{k}}=\Omega$, if $k>N$. Recalling that $\omega$ is a product state with respect to the $B_{j}$, and choosing matrix units $\left\{E_{r s}\right\}$ for $\left\{B_{0}, B_{1}, \cdots, B_{k}\right\}^{\prime \prime}$, where $k>N$, we have

$$
\begin{aligned}
\langle A \xi, \eta\rangle & =\Sigma\left\langle E_{r s} A_{r s} \xi, \eta\right\rangle \\
& =\Sigma\left\langle E_{r s}\left(\Omega_{p_{0}} \otimes \cdots \otimes \Omega_{p_{k}}\right), \Omega_{q_{0}} \otimes \cdots \otimes \Omega_{q_{k}}\right\rangle\left\langle A_{r s}(\Omega \otimes \Omega \otimes \cdots), \Omega \otimes \Omega \cdots\right\rangle \\
& =\Sigma\left\langle E_{r s}\left(\Omega_{p_{0}} \otimes \cdots \otimes \Omega_{p_{k}}\right), \Omega_{q_{0}} \otimes \cdots \otimes \Omega_{q_{k}}\right\rangle \omega\left(A_{r s}\right) \\
& =\Sigma\left\langle\Phi_{k}(A)\left(\Omega_{p_{0}} \otimes \cdots \otimes \Omega_{p_{k}}\right), \Omega_{q_{0}} \otimes \cdots \otimes \Omega_{q_{k}}\right\rangle \\
& =\left\langle\Phi_{k}(A) \xi, \eta\right\rangle,
\end{aligned}
$$

and we are done. 
Theorem 3.5. If $t \neq 0$ is an element of $[-\pi / 4, \pi / 4]$, then $\sigma_{t}$ is a shift of $\mathfrak{B}(\mathfrak{S})$.

Proof. Suppose $A \in \sigma_{t}^{k}(\mathfrak{B}(\mathfrak{S}))$ for all $k$. Then $A \in\left\{\Gamma_{k, t} B_{0} \Gamma_{k, t}^{*}, B_{k+2}, \cdots\right\}^{\prime \prime}$ for all $k$, by Lemma 3.3. Since $\Gamma_{k, t} B_{0} \Gamma_{k, t}^{*} \subseteq\left\{B_{0}, B_{1}, \cdots, B_{k+1}\right\}^{\prime \prime}, A_{k}=\Phi_{k+1}(A) \in$ $\Gamma_{k, t} B_{0} \Gamma_{k, t}^{*}$. Hence there is a sequence $y_{k} \in B_{0}$ such that $A=\mathrm{wk}-\lim \Gamma_{k, t} y_{k} \Gamma_{k, t}^{*}$, by the preceding lemma.

We shall show in the proposition below that for each $t \in[-\pi / 4, \pi / 4]$, the sequence $\left\{\Gamma_{k, t}^{*}\right\}$ converges strongly to an isometry $\Gamma^{*}$ in $\mathfrak{b}(\mathfrak{S}):$ moreover, if $\xi=$ $\bigotimes_{i} \Omega_{p_{i}}$ is a basis vector in $\mathfrak{H}$, then $\Gamma^{*} \xi$ is a vector of the form $\Omega \otimes \Lambda_{t}$. If also $\eta=\bigotimes_{\imath} \Omega_{q_{i}}$ and $e_{i j}^{0}$ is a matrix unit of $B_{0}$, observe that

$$
\begin{aligned}
\lim _{k}\left\langle\Gamma_{k, t} e_{i j}^{0} \Gamma_{k, t}^{*} \xi, \eta\right\rangle & =\lim _{k}\left\langle e_{\imath \jmath}^{0} \Gamma_{k, t}^{*} \xi, \Gamma_{k, t}^{*} \eta\right\rangle \\
& =\left\langle e_{\imath j}^{0} \Gamma^{*} \xi, \Gamma^{*} \eta\right\rangle \\
& =\delta_{\imath 1} \delta_{j 1}\langle\xi, \eta\rangle .
\end{aligned}
$$

Let $y_{k}=\sum a_{\imath j}^{k} e_{\imath j}^{0}$ for scalars $a_{\imath \jmath}^{k}$. Then $\left\|y_{k}\right\|=\left\|\Gamma_{k, t} y_{k} \Gamma_{k, t}^{*}\right\|=\left\|A_{k}\right\| \leqq\|A\|$, so that all $\left|a_{\imath j}^{k}\right|$ are bounded by $\|A\|$. From (4), $\lim _{k \rightarrow \infty}\left\langle\Gamma_{k, t} a_{\imath j}^{k} e_{\imath j}^{0} \Gamma_{k, t}^{*} \xi, \eta\right\rangle=0$ for $i \neq 1$ or $j \neq 1$, so

$$
\begin{aligned}
\langle A \xi, \eta\rangle & =\lim _{k \rightarrow \infty}\left\langle A_{k} \xi, \eta\right\rangle \\
& =\lim _{k \rightarrow \infty}\left\langle\Gamma_{k, t} y_{k} \Gamma_{k, t}^{*} \xi, \eta\right\rangle \\
& =\lim _{k \rightarrow \infty}\left\langle\Gamma_{k, t} a_{11}^{k} e_{11}^{0} \Gamma_{k, t}^{*} \xi, \eta\right\rangle \\
& =\lim _{k \rightarrow \infty} a_{11}^{k}\langle\xi, \eta\rangle .
\end{aligned}
$$

Hence $A$ is the scalar operator $\lambda I$, where $\lambda=\lim _{k \rightarrow \infty} a_{11}^{k}$.

Corollary. $\sigma$ is a $C(X)$-shift on $C(X, \mathfrak{B}(\mathfrak{E}))$.

Proof. Let $A \in \cap \sigma^{k}(C(X), \mathfrak{B}(\mathfrak{g}))$. Then $A(t)$ is a scalar operator for $t \neq 0$, by the theorem above. Hence, by continuity, $A(0)$ is a scalar operator, so $A \in$ $C(X)$.

Proposition 3.6. For $t \in[-\pi / 4, \pi / 4]$, the operators $\left\{\Gamma_{k, t}^{*}: k \in N\right\}$ converge strongly to an isometry $\Gamma_{t}^{*}$ on $\mathfrak{B}(\mathfrak{g})$. For any vector $\varphi \in \mathfrak{S}, \Gamma_{t}^{*} \varphi$ has the form $\Omega \otimes \varphi^{\prime}$.

Proof. Fix $t \neq 0$ in $[-\pi / 4, \pi / 4]$. Since $\left\{\Gamma_{k, t}: k \in N\right\}$ is a uniformly bounded sequence, the strong continuity will follow upon verifying that $\lim _{k \rightarrow \infty} \Gamma_{k, t}^{*} \xi$ exists for basis vectors $\xi=\Omega_{i_{0}} \otimes \Omega_{i_{1}} \otimes \Omega_{\imath_{2}} \otimes \cdots$. For $p \in N$ and $\eta=\Omega_{j_{0}} \otimes \Omega_{\jmath_{1}} \otimes \cdots$, note that 


$$
\begin{aligned}
U_{p, t}^{*} \eta & =e^{i t}\left\{\cos (t)-i \sin (t) T_{p}\right\} \eta \\
& =e^{i t}\left[\cos (t) \eta-i \sin (t)\left(\Omega_{j_{0}}^{\prime} \otimes \Omega_{\jmath_{1}}^{\prime} \otimes \cdots\right)\right],
\end{aligned}
$$

where $\Omega_{\jmath_{q}}^{\prime}=\Omega_{j_{q}}$, if $q \neq 0$, or $p ; \Omega_{\jmath_{0}}^{\prime}=\Omega_{\jmath_{p}}$; and $\Omega_{\jmath_{p}}^{\prime}=\Omega_{j_{0}}$. For $k \in N$, write $\Gamma_{k, t}^{*} \xi$ $=\sum_{j=1}^{n} \xi_{k, j}$, where $\xi_{k, j}$ is the sum of all tensors in $\Gamma_{k, t}^{*} \xi$ beginning with $\Omega_{\jmath}$. Then the $\xi_{k, j}$ are orthogonal, for $1 \leqq j \leqq n$, and $1=\sum_{j=1}^{n}\left\|\xi_{k, j}\right\|^{2}$.

Now choose $N$ so that $\Omega_{j_{k}}=\Omega$, for $k>N$. An application of (5) shows that for such $k, \xi_{k, j}$ is a linear combination of tensors of the form $\bigotimes_{s=0}^{\infty} \Omega_{j_{s}}$, where $\Omega_{j_{s}}=\Omega$ for $s>k+1$. Then, from (5) again,

$$
\begin{aligned}
U_{k+1, t}^{*} \xi_{k, 1} & =\xi_{k, 1}, \\
U_{k+1, t}^{*} \xi_{k, \jmath} & =e^{i t}\left\{\cos (t) \xi_{k, j}-i \sin (t) T_{k+1} \xi_{k, j}\right\} \\
& =e^{i t}\left\{\operatorname { c o s } \left(t\left(\xi_{k, j}-i \sin (t) \xi_{k, j}^{\prime}\right\},\right.\right.
\end{aligned}
$$

where $\xi_{k, \jmath}^{\prime}=T_{k+1} \xi_{k, j}$. Each summand of $\xi_{k, \jmath}^{\prime}$ has $\Omega$ in the first tensorand, and has $\Omega_{j}$ in the $(k+1)$ st tensorand.

It is now straightforwad to verify that

$$
\begin{aligned}
& \xi_{k+1, j}=e^{i t} \cos (t) \xi_{k, j}, \quad 1<j \leqq n, \quad \text { and } \\
& \xi_{k+1,1}=\xi_{k, 1}-i \sin (t) \sum_{j=2}^{n} \xi_{k, j}^{\prime},
\end{aligned}
$$

where the $n$ terms in (7.2) are mutually orthogonal (since the summands of each have $\Omega_{j}$ in the $(k+1)$ st tensorand). Moreover, for $j \neq 1, \lim \left\|\xi_{k+r, j}\right\|=0$, by $(7.1)$, so that

$$
\lim _{k \rightarrow \infty}\left\|\xi_{k, 1}\right\|=1
$$

Given $\varepsilon>0$, choose $k>N$ sufficiently large so that

$$
\begin{aligned}
S= & \sup _{p \in N}\left[\max _{1<\jmath \leq n}\left\{\left\|\xi_{k+p, \imath}\right\|\right\}\right] \\
& <\min \{\varepsilon / 2 \sqrt{2}(n-1), \varepsilon(1-\cos (t)) /(n-1)|\sin (t)| \sqrt{2}\} .
\end{aligned}
$$

Then for $r>s \geqq 1$,

$$
\begin{aligned}
\left\|\Gamma_{k+r, t}^{*} \xi-\Gamma_{k+s, t}^{*} \xi\right\|^{2} & =\left\|\sum_{j=1}^{n} \xi_{k+r, j}-\xi_{k+s, j}\right\|^{2} \\
& =\left\|\xi_{k+r, 1}-\xi_{k+s, 1}\right\|^{2}+\sum_{j=2}^{n}\left\|\xi_{k+r, j}-\xi_{k+s, j}\right\|^{2} \\
& \leqq\left\|\xi_{k+r, 1}-\xi_{k+s, 1}\right\|^{2}+\sum_{j=2}^{n}\left(\left\|\xi_{k+r, \jmath}\right\|+\left\|\xi_{k+s, \jmath}\right\|\right)^{2} \\
& <\left\|\xi_{k+r, 1}-\xi_{k+s, 1}\right\|^{2}+\varepsilon^{2} / 2 .
\end{aligned}
$$

But using (7.2) repeatedly, 


$$
\begin{aligned}
\xi_{k+r, 1}-\xi_{k+s, 1}= & -i e^{i t} \sin (t) \sum_{j=2}^{n} T_{k+s+1} \xi_{k+s, j}-\cdots \\
& -i e^{i t} \sin (t) \sum_{j=2}^{n} T_{k+r} \xi_{k+r-1, j} .
\end{aligned}
$$

Now using (7.1) repeatedly,

$$
\begin{aligned}
\left\|\xi_{k+r, 1}-\xi_{k+s, 1}\right\| & \leqq|\sin (t)|\left\{\sum_{j=2}^{n}\left\|\xi_{k+s, j}\right\|+\cdots+\sum_{j=2}^{n}\left\|\xi_{k+r-1, j}\right\|\right\} \\
& \leqq(n-1)|\sin (t)|\left\{S+S \cos (t)+\cdots+S \cos ^{r-s}(t)\right\} \\
& <(n-1)|\sin (t)|(S)\{1 / 1-\cos (t)\} \\
& <\varepsilon / \sqrt{2} .
\end{aligned}
$$

Combining our estimates, we have $\left\|\Gamma_{k+r, t}^{*} \xi-\Gamma_{k+s, t}^{*} \xi\right\|^{2}<(\varepsilon / \sqrt{2})^{2}+\varepsilon^{2} / 2=\varepsilon^{2}$, so that $\left\{\Gamma_{k, t}^{*}\right\}$ converges strongly on the basis vectors.

Now, if $\xi, \eta$ are arbitrary (orthogonal) basis vectors, $\left\langle\Gamma_{t}^{*} \xi, \Gamma_{t}^{*} \eta\right\rangle=$ $\lim _{k \rightarrow \infty}\left\langle\Gamma_{k, t}^{*} \xi, \Gamma_{k, t}^{*} \eta\right\rangle=\langle\xi, \eta\rangle$. Using this identity it is easy to show that $\Gamma_{t}^{*}$ is an isometry. Moreover, an application of (8) establishes the last statement of the proposition.

\section{Acknowledgements}

We are grateful to Mitch Baker and to Dana Williams for helpful comments and conversations. We also wish to thank the referee for a more elegant proof to Proposition 2.5.

\section{References}

[1] Dixmier, J., Les algèbres d'opérateurs dans l'espace hilbertien, Paris, GauthierVillars, 1969.

[2] Elliott, G., Derivations of matroid $C^{*}$-algebras, Invent. Math., 9 (1970), 253-269.

[3] Glimm, J., On a certain class of operator algebras, Trans. Amer. Math. Soc., 95 (1960), 318-340.

[4] Guichardet, M. A., Produits tensoriels infinis et représentations des relations d'anticommutation, Ann. Sci. Ec. Norm. Sup., 83 (1966), 1-52.

[5] Jones, V.F.R., Index for subfactors, Invent. Math., 72 (1983), 1-25.

[6] Kadison, R. V. and J. R. Ringrose, Derivations and automorphisms of operator algebras, Commun. Math. Phys., 4 (1967), 32-63.

[7] Lance, E.C., Automorphisms of certain operator algebras, Amer. Jour. Math., 91 (1969), 160-174.

[8] Phillips, J. and I. Raeburn, Automorphisms of $C^{*}$-algebras and second Cech cohomology, Indiana U. Math. Jour., 29 (1980), 799-822.

[9] - Crossed products by locally unitary automorphism groups and principal bundles, J. Oper. Thy., 11 (1984), 215-241.

[10] Powers, R.T., An index theory for semigroups of *-endomorphisms of $\mathrm{B}(\mathfrak{E})$ and type $\mathrm{II}_{1}$ factors, Can. J. Math., 40 (1988), 86-114. 
[11] Powers, R. T. and G. Price, Derivations vanishing on $S(\infty)$, Commun. Math. Phys., 84 (1982), 439-447.

[12] Price, G., Shifts of integer index on the hyperfinite $\mathrm{II}_{1}$ factor, Pac. Jour. Math., 132 (1988), 379-390.

[13] - Shifts on type $\mathrm{II}_{1}$ factors, Can. Jour. Math., 39 (1987), 492-511.

[14] Smith, M.S.B., On automorphism groups of $C^{*}$-algebras, Trans. Amer. Math. Soc., 152 (1970), 623-648. 
